\title{
Ergometria de Membros Superiores. Um Método Importante na Avaliação Cardiocirculatória ao Exercício
}

\author{
Sandra Haddad
}

São Paulo, SP

A eletrocardiografia de esforço é um método consagrado para detecção e estratificação de doença arterial coronária (DAC). A literatura da área é pródiga em trabalhos que apresentam técnicas de prescrição algorítmicas, indicações para sua utilização em diversas situações clínicas, valor prognóstico do método, bem como sua objetividade e reprodutibilidade. Na grande maioria dos artigos estudam-se as respostas ao esforço realizado em cicloergômetro ou em esteira ergométrica, sempre pressupondo que os pacientes deambulem normalmente.

A eletrocardiografia durante esforço no cicloergômetro ou na esteira, obviamente não se aplica aos portadores de afecções de membros inferiores (MMII), tais como: vasculopatias, na maioria dos casos secundária a diabetes mellitus, alterações músculo-esqueléticas, afecções reumáticas, doenças neurológicas degenerativas e nem aos portadores de deficiência física (DF), provocada por amputação de MMII ou por paraplegia.

Os portadores de deficiência física de membros inferiores (DF-MMII), tanto amputados quanto paraplégicos, realizam menor atividade física que a população sem deficiência, e até menor que os portadores de cardiopatia. A inatividade física prolongada, decorrente da perda de um ou mais elementos mecânicos necessários ao equilíbrio e à deambulação, aliada a hábitos de vida modificados e ao aumento de peso, reconhecidamente, são fatores de risco para o surgimento de doenças isquêmicas ${ }^{1}$.

De fato, embora freqüentes, as arteriopatias são de difícil diagnóstico em pessoas incapacitadas de utilizarem os MMII, pela falta de métodos apropriados para sua avaliação. Assim, nessas situações especiais, o reconhecimento dessas afecções não se realiza rotineiramente.

O objetivo deste trabalho foi demonstrar, através de revisão bibliográfica, a importância da ergometria de membros superiores (MMSS), como metodologia efetiva na avaliação da capacidade cardiocirculatória em indivíduos com limitação funcional e motora de MMII.

Associação de Assistência à Criança Defeituosa e Instituto do Coração do Hospital das Clínicas - FMUSP

Correspondência: Sandra Haddad - Incor - Div de Cardiologia Social - Av. Dr. Enéas C. Aguiar, 44 - 05403-000 - São Paulo, SP

Recebido para publicação em 4/12/96

Aceito em 2/7/97

\section{Riscos de doença arterial coronária em deficientes físicos dos membros inferiores}

Decorrentes da inatividade - Sabe-se que o paraplégico sedentário apresenta risco de amputação dos MMII, por arteriosclerose e trombose periférica, sete vezes maior que o indivíduo ativo ${ }^{1}$. Em nossa experiência, no Departamento de Cardiologia da Associação de Assistência à Criança Defeituosa (AACD), temos verificado que após um ano da cirurgia de amputação começam a ocorrer afecções coronárias ${ }^{2}$, em média, 18 meses pós-cirurgia, em cerca de $37 \%$ dos pacientes amputados de coxa uni ou bilateralmente e em $25 \%$ de paraplégicos ${ }^{3}$. Esses valores representam praticamente o dobro da incidência dessas afecções na população normal, de acordo com os dados da AACD e comparados a grupo controle de 264 indivíduos sem deficiência, da mesma faixa etária, em pacientes do ambulatório geral de funcionários do Hospital das Clínicas da FMUSP.

Decorrentes do exercício - Se a inatividade aumenta os riscos dos DF-MMII, aqueles que praticam atividade física também ficam expostos a eles, sobretudo os cardiocirculatórios decorrentes da grande sobrecarga imposta pelo trabalho de deambulação. O componente isométrico do trabalho de estabilização do corpo, realizado pela musculatura dos MMSS e do tórax, provoca elevação acentuada da pressão $\operatorname{arterial}(\mathrm{PA})^{3-8}$.

A frequiência cardíaca (FC) encontra-se elevada no início dos programas de treinamento, especialmente nos portadores de lesão medular alta (>T6), nos quais há alteração do centro de controle cardiovascular da medula ${ }^{5-9}$. Provavelmente, a sobrecarga cardiocirculatória advém do fato dos MMSS, ao suportarem o peso do corpo, estarem exercendo atividade física de intensidade mais elevada do que aquela para qual foram habitualmente preparados.

Ergometria de membros superiores - $O$ teste ergométrico (TE) de MMSS (TE-MMSS) vem merecendo atenção desde o pós-guerra e, mais recentemente, passou a ser estudado de forma mais sofisticada, com o auxílio de metodologia não invasiva mais precisa, a ergoespirometria ${ }^{10-14}$. Estudos que mostram que, além de sua utilidade na avaliação de vários parâmetros ventilatórios, como ventilação pulmonar, consumo de oxigênio, produção de dióxido de carbono e outros, é possível se obter, com algumas adapta- 
ções, o monitoramento da PA, da FC e o registro eletrocardiográfico para detecção de isquemia miocárdica, pela observação de depressão do segmento ST ${ }^{15-18}$.

O reconhecimento de doença cardíaca isquêmica, em pacientes com arteriopatia, é importante na contra-indicação a certos procedimentos cirúrgicos, como método auxiliar nos procedimentos pós-operatórios e de fundamental importância para os DF-MMII submetidos à reabilitação física, sobretudo os candidatos à deambulação, uma vez que em alguns pacientes a angina do peito é precipitada pelo esforço com os MMSS ${ }^{15}$.

Lazarus e $\mathrm{col}^{14}$, estudando a reprodutibilidade do método, não verificaram diferenças nas respostas pressóricas e cronotrópicas na repetição do mesmo TE-MMSS no mesmo dia e nos mesmos indivíduos. Quanto à validade para detecção de isquemia miocárdica em portadores de DAC, confirmada por cateterismo cardíaco, encontrou-se um r= 0,95 nos TE-MMSS, em indivíduos sem deficiência de MMII.

Comparação entre teste ergométrico de membros superiores e inferiores - Estudo comparativo entre TE realizado com MMSS e MMII demonstram que, para os mesmos níveis de trabalho, o débito cardíaco (DC) pode ser semelhante entre os dois métodos em cargas baixas; enquanto no TE-MMSS são maiores o duplo produto (DP), a ventilação pulmonar $\left(\mathrm{V}_{\mathrm{E}}\right)$, o consumo de oxigênio $\left(\mathrm{VO}_{2}\right)$ e a concentração de lactato, no TE-MMSS são menores apenas o volume sistólico (VS) e o limiar anaeróbio (LAn) expresso em porcentual da capacidade aeróbia ${ }^{12,19,20}$.

O DC pode se apresentar aumentado ou diminuído para a mesma carga no TE-MMSS em relação ao TE-MMII. O diferencial está na postura e na intensidade dos exercícios. Porém, o valor para os MMSS é plenamente maior quando é expresso em unidade de massa muscular ativa. Em conseqüência, as pressões arteriais sistólica e diastólica são maiores para membros superiores, observação feita em trabalhos mais intensos ${ }^{12,20-22}$.

A posição do exercício interfere diretamente no DC. Na posição sentada, têm-se encontrado menores valores de DC para MMSS em relação aos MMII, enquanto que, na posição supina, estes valores se equivalem. Quando se trata do valor de pico, o DC atingido no TE-MMSS sempre é menor do que no TE-MMII ${ }^{20,21,23}$.

No TE-MMSS a resistência periférica é mais elevada que no TE-MMII. Dois fatores contribuem para maior resistência periférica durante exercícios com MMSS: a maior secreção relativa de catecolaminas e a contração isométrica necessária para estabilização do tronco. Nos lesados medulares, além dos fatores mencionados, a resistência periférica torna-se ainda maior devido à tendência a hemoconcentração ${ }^{2,8,12}$.

No TE-MMSS, a FC em cargas submáximas é mais elevada, sugerindo maior estímulo simpático. Entretanto, por causa das diferenças no enchimento cardíaco, os índices de contratilidade cardíaca são maiores no TE-MMII ${ }^{24}$.

Embora níveis de lactato sangüíneo sejam semelhantes em uma mesma intensidade relativa de exercício com
MMII e MMSS, o LAn tende a ser menor no TE-MMSS. A reduzida massa muscular ativa faz com que se verifiquem menores concentrações de lactado durante esforço máximo no exercício com MMSS ${ }^{21}$.

$\mathrm{A} \mathrm{V}_{\mathrm{E}}$, para qualquer carga de trabalho, é maior com MMSS do que com MMII. Possíveis fatores que contribuem para essa resposta são: o baixo VO2máx atingido em exercícios com MMSS, a elevada concentração de lactato sangüíneo, a contração muscular isométrica proporcionalmente maior e o incremento da atividade neurogênica proveniente da musculatura ativa dos MMSS ${ }^{25}$.

Sawka ${ }^{25}$ comparou o impacto imediato de exercício de MMSS e MMII sobre a função cardiorrespiratória em DFe sem deficiência. Diferenças substanciais de resposta aguda ao exercício foram observadas nitidamente em vários parâmetros entre os dois tipos de atividade, comparados num mesmo $\mathrm{VO}_{2}$ absoluto. Muitas dessas diferenças desapareceram, quando os dados foram examinados na mesma fração dos respectivos VO2máx dos membros.

O VO2máx no exercício com MMSS, mesmo em indivíduos treinados, em geral, atinge valores mais baixos do que os conseguidos com MMII. Astrande Saltin ${ }^{24}$ identificaram valores percentuais mais altos de VO2máx para MMSS em relação aos MMII, porém, a maioria dos autores tem verificado cifras em torno de $50 \%$ a $80 \%$ do VO2máx em indivíduos sedentários e treinados 2,16,18,26,27.

Vale lembrar que, enquanto no exercício com MMII a capacidade de trabalho é limitada, principalmente, pela função cardiorrespiratória central e pelo transporte de oxigênio, no exercício com MMSS é devido a pequena massa muscular ativa e, portanto, funções como $\mathrm{V}_{\mathrm{E}}, \mathrm{FC}$, VS, DC não atingirão, habitualmente, o nível máximo verificado em exercícios de MMII.

Efeito do treinamento físico com membros superiores - Estudos prévios têm demonstrado as características de adaptação cardiocirculatória, respiratória e metabólica ao treinamento físico (TF) específico de MMSS em indivíduos jovens e saudáveis ${ }^{2,6,12,17,19}$. Essas adaptações são predominantemente periféricas e, por isso, altamente específicas do grupo muscular treinado.

Têm sido demonstrados os efeitos do TF de MMSS na melhora da aptidão cardiorrespiratória, metabólica e da função periférica de musculatura. Hickson e Rosenkoetter ${ }^{28}$ encontraram incrementos de $14 \%$ no DC e $11 \%$ no VS, enquanto a diferença arteriovenosa de $\mathrm{O}_{2}$ aumentou $16 \%$. Por outro lado, Magel e $\mathrm{col}^{23}$, após programa de 10 semanas de treinamento em jovens, não encontraram qualquer melhora do DC e do VS no exercício máximo nem no exercício submáximo, porém notaram significante diminuição da FC, aumento do VS e do trabalho total em cargas submáximas.

Alterações em parâmetros circulatórios centrais e periféricos foram comparados por Clausen e $\mathrm{col}^{4}$, utilizando metodologia invasiva antes e após treinamento de alta e moderada intensidade de MMSS e MMII, respectivamente. Foram observados aumento do VO2máx (9,9 a 15,9\%), e do trabalho total no grupo de treinamento de alta intensidade. 
No grupo que treinou em intensidade moderada não foram encontradas modificações significativas do VO2máx, do trabalho total e da resistência vascular periférica total. Nas duas intensidades de treinamento verificou-se diminuição significativa da FC em repouso.

O TF de MMSS em DF de MMII e os seus efeitos tem sido relatado em literatura ${ }^{29,30}$. Várias publicações têm demonstrado adaptações cadiorrespiratórias benéficas nesses indivíduos quando submetidos a TF de MMSS ${ }^{29-33}$.

Knutsson e $\mathrm{col}^{32}$ estudaram os efeitos do TF de MMSS em indivíduos paraplégicos. Eles realizaram de quatro a cinco sessões de TF com duração total de seis semanas. Nos DF com lesão abaixo da $6^{\mathrm{a}}$ vértebra torácica aumentou a capacidade de trabalho físico em $50 \%$. Ao contrário, nos indivíduos com lesão entre a $5^{\mathrm{a}}$ vértebra cervical e a $6^{\mathrm{a}}$ torácica, houve pouca ou nenhuma melhora em resposta ao programa de $\mathrm{TF}$.

Pollock e $\mathrm{col}^{34}$ submeteram oito DF sedentários e 11 homens sem DF a um programa de TF de endurance. A intensidade dos exercícios variou de $80 \%$ a $85 \%$ da FC máxima, 30min por sessão, três vezes por semana e duração de 20 semanas. Ambos os grupos demonstraram melhora significativa da função cardiorrespiratória. O VO2máx aumentou $39 \%$ nos indivíduos sem deficiência física, enquanto nos DF, a melhora foi de $19 \%$.

Dreisinger ${ }^{35}$ examinou os efeitos da prescrição de TF sobre a resposta cardiorrespiratória em indivíduos confinados em cadeiras de rodas. Vinte e oito homens e mulheres foram divididos em dois grupos, experimental e controle. $\mathrm{O}$ grupo experimental exercitou-se por 20min cada sessão, três vezes por semana por um período de oito semanas de TF. $\mathrm{O}$ grupo controle manteve apenas sua atividade habitual. Ao final do estudo, verificou que só houve aumento na carga máxima de trabalho e no VO2máx dos indivíduos que treinaram no grupo experimental.

Di Carlo ${ }^{36}$ utilizou um programa de TF de endurance para melhorar a capacidade cardiorrespiratória ao exercício submáximo e máximo em um indivíduo masculino de 24 anos de idade com quadriplegia. OTF consistiu de exercício em um cicloergômetro de MMSS, três vezes por semana por um período de oito semanas. A duração de cada sessão variou de 15 a 30min numa intensidade média de $80 \%$ da FC máxima. Os resultados verificados após o término do TF revelaram diminuição significativa da FC nas cargas submáximas de trabalho. A capacidade máxima de exercício e a potência aeróbica aumentaram $37 \%$ ( 35 vs $47 \mathrm{w}$ ) e $55 \%$ (11 vs $17 \mathrm{mlo}_{2}$ I $\left.\mathrm{kg}^{-1} / \mathrm{min}^{-1}\right)$, respectivamente.

Em outro estudo, Di Carlo e $\mathrm{col}^{31}$, acompanhou a resposta evolutiva de TF sobre o sistema cardiorrespiratório, durante cinco semanas em quatro indivíduos DF. Cada sessão durou $37 \mathrm{~min}$, era realizada três vezes por semana a uma intensidade entre $60 \%$ e $80 \%$ da FC máxima. Os resultados verificados antes e após o TF de MMSS, demonstraram aumentos de $55 \%$ na carga máxima de trabalho e $65 \%$ no VO2máx atingido.

Em resumo, os estudos sobre o TF de MMSS em indivíduos jovens e saudáveis, como também em DF, demons- tram claramente, que os efeitos ocorrem de maneira favorável sobre os sistema cardiorrespiratório, metabólico e muscular. Em sua grande maioria, eles têm verificado no exercício submáximo, a diminuição da FC, do quociente respiratório, da ventilação pulmonar e do consumo de oxigênio. Enquanto no exercício máximo, observam-se aumento da carga máxima de trabalho, do consumo máximo de oxigênio, da ventilação pulmonar e do ácido lático.

Em pacientes cardíacos, o TF de MMSS não é tradicional. Entretanto, na atualidade, vários estudos têm demonstrado, segurança e eficiência equivalentes ao TF realizado com MMII nesses pacientes.

Fardy e $\mathrm{col}^{37}$ estudaram as respostas eletrocardiográficas e hemodinâmicas para MMSS, MMII e combinados em 20 indivíduos cardíacos e não encontraram diferenças significativas na FC, pressão arterial sistólica (PAS) e DP nos três tipos de exercícios. A pressão diastólica média foi significativamente maior durante o exercício realizado com os MMSS, sugerindo um maior componente isométrico. A resposta eletrocardiográfica não mostrou prevalência de arritmias no exercício de MMSS, quando comparado ao esforço realizado com os MMII e combinado, respectivamente. Resultados estes confirmados por outros estudos em pacientes cardíacos ${ }^{48-50}$.

Thompson e $\mathrm{col}^{38}$ examinaram a resposta cardiorrespiratória de quatro homens treinados e oito não-treinados, com angina de peito antes e após oito semanas de TF de MMSS e MMII, respectivamente. Os indivíduos treinaram 40min em cada sessão, três vezes por semana numa intensidade próxima ou no limiar de angina. O tempo de início de angina aumentou $3,6 \mathrm{~min}$ para o grupo treinado e 1,6min para os não-treinados. ODP não modificou-se com o treinamento. Ogrupo treinado com MMSS, aumentou o VO2máx em 19\% enquanto o treinado com MMII aumentou somente $10 \%$.

Wrisley e $\mathrm{col}^{39}$ estudaram a treinabilidade de MMSS e MMII em 13 indivíduos após sofrerem infarto do miocárdio e investigaram, se as respostas do treinamento com MMSS eram semelhantes aos efeitos verificados com os MMII. Sessões de treinamento em círculo com duração de $30 \mathrm{~min}$, três vezes por semana por um período de seis semanas a uma intensidade entre $60 \%$ e $80 \%$ do VO2máx foram realizadas. Os indivíduos alternaram os equipamentos que incluíam esteira, ergômetro de MMSS e MMII, remoergômetro modificado exercícios em um banco. Os resultados demonstraram diminuição da FC, PAS e percepção subjetiva ao esforço em cargas submáximas realizadas na esteira e no ergômetro de MMS, respectivamente. Enquanto isso, o VO2máx e a carga máxima atingida aumentaram $13 \%$ e $11 \%$ e $24 \%$ e $23 \%$ no exercício realizado com MMSS e MMII, respectivamente.

Os resultados indicaram que a melhora qualitativa e quantitativa da capacidade cardiorrespiratória pelo exercício aeróbio realizado com os MMSS, é comparativamente a mesma observada com os MMII. Portanto, os resultados dessas pesquisas, são encorajadores e sugerem que o TF de MMSS é um importante meio para melhorar o nível de aptidão física de indivíduos com limitações de MMII e/ou daqueles que realizam atividades ocupacionais de MMSS. 


\section{Técnicas para realização do teste ergométrico de membros superiores}

Escolha do ergômetro - Na AACD utilizamos, basicamente, dois tipos de ergômetros, o cicloergômetro adaptado para MMSS e o ergômetro de manivela. De acordo com a meta a ser atingida, varia a escolha do ergômetro. O cicloergômetro adaptado para MMSS propicia maiores incrementos no desempenho muscular aeróbio e tem ampla aplicação na avaliação física funcional de MMSS (cardiocirculatória, capacidade aeróbia muscular, etc) ${ }^{25-29}$. É um ergômetro de baixo custo e de fácil utilização na avaliação e prescrição de nossos pacientes ${ }^{40}$. A diferença básica dos dois tipos de ergômetros está na engrenagem e circunferência do volante. No ergômetro adaptado Monark, elas foram dimensionadas de modo que uma volta completa dos pedais desenvolva uma distância teórica comparável a $6 \mathrm{~m}$, enquanto no ergômetro de manivela, a distância é de $3 \mathrm{~m}$. A escala de carga de trabalho, no ergômetro adaptado Monark, é graduada em kilopounds (kp) (1 kp é a força que age sobre uma massa de $1 \mathrm{~kg}$ à aceleração normal da gravidade). O poder de frenagem $(\mathrm{kp})$ estipulado pelo ajuste da tensão da correia, multiplicado pela distância pedalada ou ciclada $(\mathrm{m})$, fornece o volume de trabalho em kp/m $(\mathrm{kpm})$ ou kilograma $/ \mathrm{m}(\mathrm{kgm})$. Se a distância for expressa pelo tempo gasto na realização do exercício, então, obtém-se a média do trabalho em $\mathrm{kpm} / \mathrm{min}^{-1}$ ou $\mathrm{kgm} / \mathrm{min}^{-141}$.

O envolvimento de pequenos grupos musculares provoca fadiga muscular precoce, principalmente em DF do sexo feminino, sedentárias. A ocorrência de fadiga precoce é menos acentuada em indivíduos treinados em modalidades esportivas que requeiram grande participação dos MMSS ( natação, caiaque, remo, etc). Para pessoas de baixa aptidão física, principalmente do sexo feminino, diabéticas, preferimos utilizar inicialmente o ergômetro de manivela, que mobiliza a musculatura de braços e antebraços, poupando os músculos do tronco e facilitando a realização de cargas baixas por maior período de tempo.

Quando o objetivo é obter resposta rápida e expressiva do sistema cardiocirculatório sempre utilizamos o cicloergômetro adaptado para MMSS, uma vez que permite execução de níveis elevados de trabalho, aumentando a FC > 85\% da FC máxima predita, por envolver maior número de grupos musculares (cintura escapular, tronco), e atingir maior VO2máx com menores níveis de cansaço e lactacidemia ${ }^{42}$.

Protocolo - Na AACD, utilizamos protocolos intermitentes com incrementos de cargas de trabalho escalonados de 125 (20w) e $140 \mathrm{kgm} / \mathrm{min}^{-1}(25 \mathrm{w})$ para mulheres e homens, respectivamente no cicloergômetro adaptado Monark e ergômetro de manivela para MMSS. Cada estágio tem duração de 3 min intercalados com $1 \mathrm{~min}$ de intervalo ativo. O intervalo entre as cargas tem duração de 30s de para medição da PA e registro de eletrocardiograma seguidos por mais $30 \mathrm{~s}$ de repouso com os mãos fora dos pedais adaptados e apoiadas sobre a mesa. Para adequada medição da PA durante o intervalo ativo, um dos braços permanece em movimento, enquanto a PA é medida no membro contralateral por método auscultatório, na artéria braquial. São realizados dois registros eletrocardiográficos: $\mathrm{o} 1^{\circ}$ durante o intervalo ativo e o $2^{\circ}$ imediatamente após à soltura dos pedais adaptados.

Utilizamos velocidades entre 69 a 83 rpm para mulheres e 83 a 95rpm para homens, respectivamente. Essas rotações são ajustadas para ambos os sexos e têm sido utilizadas com relativa facilidade ao longo de cinco anos em cerca de 800 TE-MMSS, possibilitando melhor eficiência muscular, resposta cronotrópica satisfatória atingindo a FC submáxima predita para a idade, semelhantes aos achados de Hardison $\mathrm{e} \mathrm{col}^{43}$. Velocidades superiores às estabelecidas provocam interrupção precoce por fadiga muscular localizada, enquanto inferiores tornam os testes demasiadamente longos e sem eficácia.

Concluindo, a ergometria de MMSS ainda pouco conhecida e utilizada pela cardiologia, tem sido de grande utilidade como meio alternativo na avaliação de parâmetros cardiocirculatórios em indivíduos com limitações provocadas por alterações de ordem motora, vascular e/ou neurologica.

O método tem permitido constatar, na maioria dos casos, a verificação de alterações cardiovasculares importantes, como também, os efeitos de treinamento físico (TF). Estudos em indivíduos com limitação motora de MMII indicam que o TF aeróbio de MMSS realizado por várias semanas, podem aumentar significativamente a potência musculare o VO2máx ${ }^{31-33}$.

Embora o TF de MMSS apresente um limite inferior para o aumento da potência aeróbia quando comparado ao TF com MMII, ele pode ser atingido. Todavia, a magnitude deste aumento parece ser dependente do nível inicial de aptidão física e do tamanho da massa muscular envolvida no exercício.

Geralmente, os maiores ganhos são observados em indivíduos com baixa capacidade física ${ }^{44}$. As adaptações fisiológicas provenientes do TF aeróbio com MMSS são devidas a melhorias periféricas (melhora da densidade capilar e/ou capacidade metabólica dentro dos músculos) mais que adaptações circulatórias centrais ${ }^{23,44}$

Alguns estudos ${ }^{14-16,18,45}$ têm demonstrado, que o método empregado é reprodutível e de grande utilidade como teste diagnóstico na detecção de isquemia miocárdica via depressão do segmento ST e/ou angina de peito provocada pelo exercício de MMSS ${ }^{15,46,47}$.

Assim, consideramos a ergometria de MMSS uma alternativa importante para avaliação funcional de DF, daqueles acometidos por quaisquer afecções dos MMII que dificultem a deambulação e em indivíduos que realizam atividades físicas ocupacionais dominantes de MMSS. 


\section{Referências}

1. Yekutiel M, Brooks ME, Ohry A et al - The prevalence of hypertension ischaemic heart disease and diabetes in traumatic spinal cord injuried patients and amputees. Paraplegia 1989; 27: 58-62.

2. Haddad S, Silva PRS, Ferraretto I - Análise descritiva da incidência de eventos mórbidos em deficiente físico. Arq Bras Cardiol 1992; 59(supl II): 154.

3. Haddad S, Silva PRS, Ferraretto I - Assimilação compensatória de treinamento físico aeróbio de membros superiores. Arq Bras Cardiol 1992; 59(supl II): 158.

4. Clausen JP, Klausen B, Rasmussen B et al - Central and peripheral circulatory changes after training of the arms or legs. Am J Physiol 1973; 225: 675-82.

5. Mathias CJ, Frankel HL - Cardiovascular control em spinal man. Ann Rev Physiol 1989; 50: 577-92.

6. Glaser RM - Arm exercise training for wheelchair users. Med Sci Sports Exer 1989; 21(suppl S): S149-S57.

7. Patterson R, Fisher I - Cardiovascular stress of crutch waking. Arch Phys Med Rehabil 1981; 62: 257-26.

8. Hanson P, Nagle F - Os exercícios, e o coração: exercício isométrico. Respostas cardiovasculares nas populações normal e cardíaca. In: Clinicas Cardiológicas, vol 2. São Paulo: Interlivros, 1987.

9. Kivowitz C,Parmley WN, Donoso Ret al-Effects of isometric exercice on cardiac performance. The grip test. Circulation 1971; 44: 994-1002.

10. Asmussen E, Nielsen M - Regulation of body temperature during work performed with arms and legs. Acta Physiol Scand 1945; 14: 373-83.

11. Asmussen E, Hemmingsen I - Determination of maximum capacity at different ages. J Clinical Lab Investigation 1947; 10: 67-71.

12. Bevergard S, Freyschuss U, Strandell T - Circulatory adaptation to arm and leg exercise in supine and sitting position. J Appl Physiol 1966; 21: 37-46.

13. Frankel HL, Michaelis LS, Golding DR et al - The blood pressure in paraplegia Paraplegia 1972; 10:193-8.

14. Lazarus B, Cullinane E, Thompson PD - Comparison of the results and reprodutibility of arm and leg exercise tests in men with angina pectoris. Am J Cardiol 1981; 47: 1075-9.

15. Shaw DJ, Crawford MH, Karliner JS et al - Arm crank ergometry: A new method for the evolution of coronary artery disease. Am J Cardiol 1974; 33: 801-5.

16. Bar-Or O,Zweren LD - Maximal oxygen consumption test during arm exercisereliability and validity. J Appl Physiol 1975; 38: 424-6.

17. Loftin $\mathrm{M}$ - Effect of arm training on central and peripheral circulatory function. Med Sci Sports Exerc 1988; 20: 136-41.

18. Eriksson P, Loftrom L, Ekblom B - Aerobic power during maximal exercise in untrained and well-trained persons with quadriplegia and paraplegia. Scand J Rehab Med 1988; 20: 141-7.

19. Vokac Z, Bell H, Bantz-Holter E et al - Oxygen uptake, heart rate relationship in leg and arm exercice, sitting and standing. J Appl Physiol 1975; 39: 54-9.

20. Stenberg J, Astrand PO, Ekblom B et al - Hemodynamic response to work with different muscles groups, sitting and supine. J Appl Physiol 1967; 22: 61-70.

21. Miles DS, Sawka MN, Hanpeter DEet al -Central hemodynamics during progressive upper and lower body exercise and recovery. J Appl Physiol 1984; 57: 36670 .

22. Pimental N, Sawka MN, Billings DS et al - Physiological responses to prolonged upper body exercise. Med Sci Sports Exerc 1984; 16: 360-5.

23. Magel JE, McArdle WD, Toner Met al - Metabolic and cardiovascular adjustment to arm training. J Appl Physiol 1978; 45: 75-9.

24. Astrand PO, Saltin B - Maximal oxygen uptake and heart rate in various types of muscular activity. J Appl Physiol 1961; 16: 977-81.
25. Sawka MN - Physiology of upper body exercise. Exercise Sports Science Reviews 1986; 14: 175-221.

26. Bobbert AC - Physiolgical comparisons of three types of ergometry. J Appl Physiol 1960; 41: 1007-14

27. Seals DR $-\mathrm{VO}_{2 \max }$ and variable type exercise among well-trained upper body ath letes. Res Q Exerc Sport 1982; 53: 58-63.

28. Hickson RC, Rosenkoetter MA - Strenght training effect on aerobic power and short-term endurance. Med Sci Sports Exerc 1981; 13: 17-20.

29. Davis JA, Vodak P, Wilmore JHet al - Anaerobic threshold and maximal aerobic power for three modes of exercise. J Appl Physiol 1976; 41: 544-50.

30. Dreisinger TE, Londeree BR - Wheelchair exercise: A review. Paraplegia 1982 20: $20-34$

31. Di Carlo SE, supp MD, Taylor HC - Effect of arm ergometry training on physical work capacity of individuals with spinal cord injuries. Phys Ther 1983; 63 1104-7.

32. Knutsson EE, Lewenhaupt-Olsson E, Thorsen M - Physical work capacity and physical conditioning in paraplegic patients. Paralegia 1973; 11: 205-216.

33. Miles DS, Sawka MN, Wilde SW et al - Pulmonary function changes in wheelchair athletes subsequent to exercise training. Ergonomics 1982; 25: 239-46.

34. Pollock ML, Miller HS, Linnerud AC et al - Arm pedaling as an endurance training regimen for the disabled. Arch Phys Med Rehabil 1974; 55: 418-24.

35. Dreisinger TE - Wheelchair ergometry. A training study. Unpublished doctoral dissertation. Columbia: University of Missouri, USA, 1978

36. Di Carlo SE - Improved cardiopulmonary status after a two-month program of graded arm exercise in a pacient with C6 quadriplegia. Phys Ther 1982; 62: 456-

37. Fardy PS, Doll NE, Reitz L et al - Prevalence of dysrhythmias during upper, lower and combined upper and lower extremity exercise in cardiac patients. Med Sci Sports Exer 1981; 13: 137

38. Thompson PD, Cullinae E, Lazarus B et al - Effect of exercise training on the untrained limb exercise performance of men with angina pectoris. Am J Cardiol 1981a; 48: 844-50.

39. Wrisley D, Franklin B, Vander L et al - Effects of exercise training on arm and leg aerobic capacity in cardiac patients. Med Sci Sports Exer 1983; 15: 92.

40. Franklin BA - Exercise testing, training and arm ergometry. Sports Med 1985; 2 100-19.

41. Astrand PO - Testes com a ergométrica e manual de operações da bicicleta mecânica monark. São Paulo: Bicicletas Monark SA.

42. Louhevaara V, Sovijarvi A, Ilmarinen J et al - Differences in cardiorespiratory reponses during and after arm cranck and cycle exercise. Acta Physiol Scand 1990; 138: 133-43.

43. Hardison GT, Israel RG, Somes GW - Physiological responses to different cranking rates during submaximal arm ergometry in paraplegic males. Adapt Phys Activity Quarterly 1987; 4: 94-105.

44. Glaser RM, Davis GM - Wheelchair-dependent individuals. In: Franklin BA Gordon S, Timms GC - Execise in Modern Medicine: Testting and Prescription in Health and Disease, eds. Baltimore: Williams \& Wilkins, 1988; 237-67.

45. Sawka MN, Foley ME, Pimentel NA et al - Determination of maximal aerobic power during upper-exercise. J Appl Physiol 1983; 54: 113-17.

46. Balady GJ, Weiner DA, McCabe CHet al - Value of arm exercise testing in detecting coronary artery disease. Am J Cardiol 1985; 55: 37-9.

47. Schawade J, Blomqvist CG, Shapiro W - A comparison of the response to arm and leg work in patients with ischemic heart disease. Am Heart J 1977; 94: 354-9. 